Paper No. 211-7

Presentation Time: 3:20 PM

\title{
ORIGIN AND EVOLUTI ON OF DWARF PLANET CERES FROM DAWN
}

RAYMOND, Carol A. ${ }^{1}$, RUSSELL, C.T. ${ }^{2}$, AMMANNITO, E. ${ }^{3}$, BUCZKOWSKI, D.L. ${ }^{4}$, CASTILLO-ROGEZ, J.C..$^{1}$, COMBE, J-P. ${ }^{5}$, DE SANCTIS, M.C. ${ }^{6}$, JAUMANN, R. ${ }^{7}$, MCCORD, T.B. ${ }^{5}$, MCSWEEN, H.Y. ${ }^{8}$, NATHUES, A. ${ }^{9}$, PRETTYMAN, T.H. ${ }^{10}$ and SCHENK, P.M. ${ }^{11}$, (1)Jet Propulsion Laboratory, California Institute of Technology, Pasadena, CA 91109, (2)Earth, Planetary and Space Sciences/IGPP, University of California, Los Angeles, 603 Charles Young Drive, 3845, Los Angeles, CA 90095, (3)EPSS-IGPP, UCLA, 595 Charles Young Drive East, Los Angeles, CA 90025, (4)Space Departrment, Johns Hopkins Applied Physics Laboratory, 11100 Johns Hopkins Rd, Laurel, MD 20723, (5)Bear Fight Institute, P.O. Box 667, 22 Fiddler's Rd, Winthrop, WA 98862, (6)INAF, Instituto di Astrofisica Spaziale e Fisica Cosmica, Rome, Italy, (7)German Aerospace Center (DLR) Berlin, Institute of Planetary Research, Rutherfordstrasse 2, Berlin, D-12489, Germany, (8)Department of Earth and Planetary Sciences, University of Tennessee, 1412 Circle Drive, University of Tennessee, Knoxville, TN 37996-1410, (9)Max-Planck Institute for Solar System Research, Katlenburg-Lindau, Germany, (10)Planetary Science Institute, Los Ranchos de Albuquerque, NM 87107, (11)Lunar and Planetary Institute, Universities Space Research Association, 3600 Bay Area Boulevard, Houston, TX 77058, carol.a.raymond@jpl.nasa.gov

Prior to Dawn's arrival, Ceres was already known from ground- and space-based observations to be a dark, wet dwarf planet with evidence for altered minerals and water vapor emissions. Dawn found a very dark, cratered surface punctuated by small extremely bright areas. Contrary to the expectation of an ice-rich, viscously-relaxed smooth surface resulting from physical differentiation and freezing of an ancient subsurface ocean, its surface has many craters, implying a mechanically strong thick crust. Ceres is, however, missing the largest expected craters and is gravitationally relaxed at lowest orders, implying that the strong crust overlies a weaker deep interior. Dark material, phyllosilicates, ammoniated clays, and carbonates dominate Ceres' surface. The ubiquitous presence of ammoniated minerals suggests formation in a cold environment, pointing to an outer solar system origin. The distribution of minerals indicates that Ceres' interior experienced pervasive alteration. Moreover, certain processes that are not yet fully understood resulted in material formed at depth being brought to the surface. Water ice has also been observed in fresh craters at high latitudes, and elemental measurements indicate the presence of water ice in the immediate subsurface. The topography and morphology of the surface reveal regional variations, with smoother, apparently resurfaced areas that are generally at lower elevation and rougher areas with greater relief. Local morphology such as crater floors deposits, isolated mountains, and the enigmatic bright areas indicate active processes on Ceres that likely involves brine-driven cryovolcanism.

\section{Session No. 211}

P3. Exploring the Third Zone: The Geology of Pluto, Charon, and the Kuiper Belt

Tuesday, 27 September 2016: 1:30 PM-5:30 PM

Mile High Ballroom 2A/3A (Colorado Convention Center)

Geological Society of America Abstracts with Programs. Vol. 48, No. 7 doi: 10.1130/abs/2016AM-282184

(C) Copyright 2016 The Geological Society of America (GSA), all rights reserved. Permission is hereby granted to the author(s) of this abstract to reproduce and distribute it freely, for noncommercial purposes. Permission is hereby granted to any individual scientist to download a single copy of this electronic file and reproduce up to 20 paper copies for noncommercial purposes advancing science and education, including classroom use, providing all reproductions include the complete content shown here, including the author information. All other forms of reproduction and/or transmittal are prohibited without written permission from GSA Copyright Permissions.

Back to: P3. Exploring the Third Zone: The Geology of Pluto, Charon, and the Kuiper Belt 